\title{
Palliative Care for the Late-Stage Cancer Patient During the COVID-19 Era: A Report of the Challenges
}

\author{
Javier Alejandro Millanes-Galaz, Evelyn Dayanna Otero-Torres and Diana Evelyn Villa-Guillen* \\ Department of Foreign Languages, Graduate Studies and Research, University Kino A.C.,Mexico
}

\begin{abstract}
Objective: To report the main factors of oncological palliative care in the late-stage cancer patient affected during the pandemic of COVID-19, as recognized by the international medical community.
\end{abstract}

Methods: We followed the PRISMA guidelines and searched on the electronic database PubMed using the search terms: palliative care, neoplasms, cancer, pandemic, and quality of life. We excluded articles of recommendations and guidelines, opinion articles, conference abstracts, or articles published before March $11^{\text {th }}, 2020$, the official WHO date of the COVID-19 pandemic.

Results: Seven studies satisfied the inclusion criteria of 1,400. Those research articles are from Norway, UK, Palestine, Egypt, Jordan, Africa, India, The Netherlands, Germany, and Turkey. Three studies were cross-sectional surveys, one was observational retrospective, two were observational prospective, and one was a longitudinal cohort observational study. Factors identified by the international medical community are the overall quality of oncological palliative care services, emotional dysfunction, and mental disorders.

Conclusion: The standard palliative care for late-stage cancer patients has been affected in distinct countries. Changes in policies and guidelines, and the scarcity of resources in the health sector, have reduced the medical attention provided for the cancer patient receiving palliative care. More studies are required to identify additional needs of late-stage cancer patients during the current health crisis.

KEYWORDS: Review; Palliative Care; Late-Stage Cancer; Pandemic; COVID-19

\section{INTRODUCTION}

The pandemic of COVID-19 has affected the health sector and has displaced other services like oncological palliative care. This included postponement of most oncological care to minimize infection risk, as late-stage cancer patients (stages III, IV, metastasis) are considered a high-risk patient population Bartels [1]; Sigosrski [2]. Palliative care is one of the most affected services, understanding this one as provided for improving the quality-of-life Gupta [3]. The impact of the COVID-19 pandemic affected medical care, prioritizing treatment of those infected with SARS-CoV-2 over other patients. Medical personnel had to modify treatment and follow-up protocols, especially for cancer patients, to provide an acceptable standard of care Bakhribah [4]; Aapro [5]. Those adjustments were achieved in a short time frame, leading to clinical data exchange, recommendations, and updated guidelines for cancer management and support during the COVID-19 era Bakhribah [4]. Moreover, scare studies address this urgent need for hospital planning, with the objective to reduce the adverse effects for cancer patients Pathania [6]. The aim of this review is to report the factors of the standard oncological palliative care affected during the pandemic of COVID-19. Results from this review may provide insights regarding health care quality, its limitations, and effectiveness concerning oncological palliative care.

\begin{tabular}{|c|c|}
\hline Quick Response Code: & $\begin{array}{l}\text { Address for correspondence: Diana Evelyn Villa-Guillen, University Kino A.C. } \\
\text { Pbro. Pedro Villegas Ramírez, Casa Blanca, Mexico }\end{array}$ \\
\hline 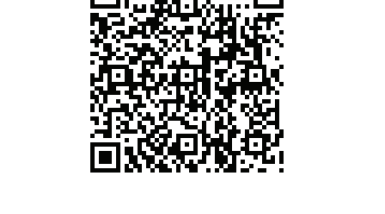 & $\begin{array}{l}\text { Received: August 28, } 2021 \text { Published: September 17, } 2021 \\
\text { How to cite this article: Javier AMG, Evelyn DOT, Diana EVG. Palliative Care for the Late- } \\
\text { Stage Cancer Patient During the COVID-19 Era: A Report of the Challenges. 2021- 3(5) OAJBS. } \\
\text { ID.000318. DOI: } 10.38125 / \text { OAJBS.000318 }\end{array}$ \\
\hline
\end{tabular}




\section{METHODS}

We conducted a review and followed the protocol detailed in the Preferred Reporting Items for Systematic Reviews and MetaAnalyses (PRISMA) statement. To identify gaps in knowledge, we conducted a scoping review instead of a systematic review.

\section{Search Strategy}

We conducted a systematic search at PubMed using the following terms: 'palliative care', 'neoplasms', 'cancer', 'pandemic', and 'quality of life'. Moreover, we used the following MeSH terms to ensure a comprehensive review of the evidence: ("palliative care"[MeSH Terms] OR ("palliative"[All Fields] AND "care"[All Fields]) OR "palliative care"[All Fields]) AND ("neoplasms"[MeSH Terms] OR "neoplasms"[All Fields] OR "cancer"[All Fields]) AND ("pandemics"[MeSH Terms] OR "pandemics"[All Fields] OR "pandemic"[All Fields]) AND ("quality of life"[MeSH Terms] OR ("quality"[All Fields] AND "life"[All Fields]) OR "quality of life"[All Fields]) AND (“2018/03/11"[PubDate]: “2021/04/17”[PubDate]).

The search was restricted to scientific publications in English and Spanish from March $11^{\text {th }} 2020$, to March $17^{\text {th }}, 2021$. The initial date was chosen due to being the official one of the WHO announcements for the pandemic of COVID-19.

\section{Eligibility Criteria}

Inclusion criteria: Three reviewers performed the study selection. The reviewers included the study if it focused on palliative care related to (a) cancer patients at stage III, IV or metastasis, (b) health care infrastructure, (d) health care providers and staff, (e) psychological analysis of health care professionals or (f) that of cancer patients during the pandemic of COVID-19.

Exclusion criteria: Studies not included were those not related to oncological palliative care; or aimed for palliative care but fell in any of the following:

(a) The study was published before the official date of the pandemic.

(b) The article was not focused on cancer patients but on COVID-19 patients.

(c) The study involved the use of telemedicine for nononcological palliative care.

(e) The study was a summary of guidelines and recommendations.

(f) The publication was that of conferences or abstracts with no quantitative data.

(g) The publication was an opinion article or a letter to the editor

(h) The overall quality of the article was critical or moderate as defined by the online Cochrane tool Risk of Bias in Non-randomized Studies of Interventions (ROBINS-I); Sterne [7].

Reviewers removed duplicates and evaluated full-text articles to determine compliance with the inclusion criteria. The authors solved disagreements through discussion until reaching a consensus.

Definitions and outcomes: For the late-stage cancer patient, the definition used was that of the American Joint Committee on Cancer staging criteria Amin [8]. For oncological palliative care, it was used the WHO definition, which considers it as an approach not intended to cure the patient but to offer an acceptable quality of life until death, with the purpose to support the patient and their relatives towards closure WHO [9]. The outcomes for this review included the impact of the pandemic of COVID-19 on the late-stage cancer patient (anxiety and related disorders, follow-up, treatment modification or delays, relocation, health insurance), on the health care provider (anxiety and mental disorders, relocation, and reassignment of duties), and the health care infrastructure aimed for palliative care (resources, restructuration by COVID-19, among others).

\section{Overall Quality of Included Studies}

For evaluating the overall quality for each study, reviewers performed a critical appraisal using the online Cochrane tool Risk of Bias in Non-randomized Studies of Interventions (ROBINS-I) Sterne [7]. There are seven domains for ROBINS-I bias assessment. Those are D1 (bias due to confounding), D2 (selection of participants), D3 (classification of interventions), D4 (deviations from intended interventions), D5 (missing data), D6 (measurement of outcomes), and D7 (selection of reported results). All included studies were analyzed for the seven risks of bias domains.

\section{RESULTS}

\section{Article Selection Process}

The search strategy retrieved a total of 1400 articles. After inclusion criteria and duplication removal, 49 studies were eligible for the title and abstract screening. We excluded 37 articles after the full-abstract screening, leaving 12 research studies for the analysis. One study did not comply with the inclusion criteria because it was conducted before the pandemic, leaving 11 articles. After conducting bias analysis by ROBINS-I, four studies were classified with a critical/moderate overall bias and were not eligible. A total of 7 research studies matched the criteria selection for the present report.

\section{Study Characteristics and ROBINS-I Quality Assessment}

A summary of the characteristics for each included study in this review is given in Table 1 (Table 1, Appendix). The description entails country of origin, study design, factor(s) of palliative care, study sample size, reported outcome(s) related to COVID-19 pandemic, and ROBINS-I overall bias assessment. In terms of countries, one study involved international collaborations across distinct countries (UK, Palestine, Egypt, and Jordan). There were other studies conducted in Norway, Africa, The Netherlands, Germany, India and Turkey.

With regards to study design, three studies were crosssectional surveys. Three studies were observational prospective, and one was observational retrospective research. One study was a longitudinal cohort observational study. Selected studies evaluated the following factors related to palliative care: palliative care services (2 studies), general cancer care services (1 study), quality of life and emotional functioning of cancer patients ( 2 studies), anxiety and depression in cancer patients ( 1 study), and palliative radiotherapy during the last month of life (1 study). Outcomes of these studies evaluated changes of the priorly mentioned factors in response to the COVID-19 pandemic.

ROBINS-I quality assessment evaluated seven bias domains for each study (Table 2, Appendix). This report included solely studies classified with an overall low bias according to ROBINS-I. All articles were evaluated for each bias domain and depicted in Table 2. 
Table 1: Characteristics of included studies.

\begin{tabular}{|c|c|c|c|c|c|c|c|c|}
\hline $\begin{array}{c}\text { First } \\
\text { Author }\end{array}$ & $\begin{array}{c}\text { Year of } \\
\text { Publication }\end{array}$ & PMID & PMC & Country & Study Design & $\begin{array}{c}\text { Factor(s) of } \\
\text { Oncological } \\
\text { Palliative } \\
\text { Care }\end{array}$ & $\begin{array}{c}\text { Study } \\
\text { sample size } \\
\text { (n) }\end{array}$ & $\begin{array}{l}\text { Reported } \\
\text { outcome(s) } \\
\text { related to } \\
\text { COVID-19 } \\
\text { pandemic }\end{array}$ \\
\hline Nieder C & 2021 & 33402522 & PMC7880726 & Norway & $\begin{array}{l}\text { Observational } \\
\text { retrospective }\end{array}$ & $\begin{array}{c}\text { Palliative } \\
\text { radiotherapy } \\
\text { during the last } \\
\text { month of life in } \\
\text { the COVID-19 } \\
\text { scenario }\end{array}$ & 47 patients & $\begin{array}{l}\text { Changes on } \\
\text { palliative } \\
\text { radiotherapy }\end{array}$ \\
\hline $\begin{array}{c}\text { Boufkhed } \\
\text { S }\end{array}$ & 2020 & 33227380 & PMC7679234 & $\begin{array}{l}\text { UK, Palestine, } \\
\text { Egypt, Jordan }\end{array}$ & $\begin{array}{l}\text { Cross-sectional } \\
\text { online survey }\end{array}$ & $\begin{array}{l}\text { Palliative care } \\
\text { services in } \\
\text { response to } \\
\text { the COVID-19 } \\
\text { pandemic }\end{array}$ & $\begin{array}{c}43 \text { health care } \\
\text { providers and } \\
\text { staff }\end{array}$ & $\begin{array}{l}\text { Capacity of } \\
\text { palliative } \\
\text { health care } \\
\text { services }\end{array}$ \\
\hline $\begin{array}{c}\text { Boufkhed } \\
\text { S }\end{array}$ & 2020 & 32949761 & PMC7493734 & Africa & $\begin{array}{l}\text { Cross-sectional } \\
\text { online survey }\end{array}$ & $\begin{array}{l}\text { Palliative care } \\
\text { services in } \\
\text { response to } \\
\text { the COVID-19 } \\
\text { pandemic }\end{array}$ & $\begin{array}{c}83 \\
\text { respondents }\end{array}$ & $\begin{array}{l}\text { Capacity of } \\
\text { palliative } \\
\text { health care } \\
\text { services }\end{array}$ \\
\hline $\begin{array}{l}\text { Bartels } \\
\text { MMTJ }\end{array}$ & 2021 & 33634347 & PMC7906245 & $\begin{array}{l}\text { The } \\
\text { Netherlands }\end{array}$ & $\begin{array}{l}\text { Observational } \\
\text { prospective }\end{array}$ & $\begin{array}{l}\text { Quality of life } \\
\text { and emotional } \\
\text { functioning of } \\
\text { cancer patients } \\
\text { during the } \\
\text { first COVID-19 } \\
\text { lockdown }\end{array}$ & $\begin{array}{l}169 \\
\text { respondents }\end{array}$ & $\begin{array}{c}\text { Emotional } \\
\text { functioning } \\
\text { and quality of } \\
\text { life of cancer } \\
\text { patients }\end{array}$ \\
\hline Mitra M & 2020 & 32830619 & PMC7448263 & India & $\begin{array}{l}\text { Cross-sectional } \\
\text { online survey }\end{array}$ & $\begin{array}{c}\text { Cancer care } \\
\text { services during } \\
\text { the COVID-19 } \\
\text { era }\end{array}$ & $\begin{array}{c}36 \\
\text { respondents }\end{array}$ & $\begin{array}{l}\text { Challenges } \\
\text { experienced } \\
\text { by cancer } \\
\text { patients }\end{array}$ \\
\hline $\begin{array}{l}\text { Troschel } \\
\text { FM }\end{array}$ & 2021 & 33805663 & PMC7999211 & Germany & $\begin{array}{l}\text { Longitudinal } \\
\text { cohort } \\
\text { observational } \\
\text { study }\end{array}$ & $\begin{array}{l}\text { Quality of life } \\
\text { and emotional } \\
\text { functioning of } \\
\text { cancer patients } \\
\text { during the } \\
\text { first COVID-19 } \\
\text { lockdown }\end{array}$ & $\begin{array}{l}63 \text { patients } \\
\text { and } 37 \\
\text { relatives }\end{array}$ & $\begin{array}{l}\text { Quality of } \\
\text { life of cancer } \\
\text { patients and } \\
\text { their relatives }\end{array}$ \\
\hline $\begin{array}{c}\text { Yildirim } \\
\text { OA }\end{array}$ & 2021 & 33635508 & PMC7907665 & Turkey & $\begin{array}{l}\text { Observational } \\
\text { prospective }\end{array}$ & $\begin{array}{c}\text { Anxiety and } \\
\text { depression in } \\
\text { cancer patients }\end{array}$ & 595 patients & $\begin{array}{l}\text { Impact of } \\
\text { treatment } \\
\text { delays, } \\
\text { anxiety, and } \\
\text { depression } \\
\text { in cancer } \\
\text { patients }\end{array}$ \\
\hline
\end{tabular}

Table 2: ROBINS-I bias assessment of included studies.

\begin{tabular}{|c|c|c|c|c|c|c|c|c|c|c|c|}
\hline Author & $\begin{array}{c}\text { Year of } \\
\text { Publication }\end{array}$ & PMID & PMC & $\begin{array}{c}\text { Overall } \\
\text { Bias }\end{array}$ & D1 & D2 & D3 & D4 & D5 & D6 & D7 \\
\hline Nieder C & 2021 & 33402522 & PMC7880726 & LOW & MODERATE & LOW & MODERATE & LOW & LOW & LOW & LOW \\
\hline $\begin{array}{c}\text { Boufkhed } \\
\text { S }\end{array}$ & 2020 & 33227380 & PMC7679234 & LOW & LOW & LOW & LOW & LOW & LOW & LOW & LOW \\
\hline $\begin{array}{l}\text { Boufkhed } \\
\text { S }\end{array}$ & 2020 & 32949761 & PMC7493734 & LOW & LOW & LOW & LOW & LOW & LOW & MODERATE & LOW \\
\hline $\begin{array}{l}\text { Bartels } \\
\text { MMTJ }\end{array}$ & 2021 & 33634347 & PMC7906245 & LOW & LOW & LOW & LOW & LOW & LOW & LOW & LOW \\
\hline Mitra M & 2020 & 32830619 & PMC7448263 & LOW & MODERATE & LOW & MODERATE & LOW & $\mathrm{NI}$ & LOW & MODERATE \\
\hline $\begin{array}{l}\text { Troschel } \\
\text { FM }\end{array}$ & 2021 & 33805663 & PMC7999211 & LOW & LOW & LOW & LOW & LOW & LOW & LOW & LOW \\
\hline $\begin{array}{l}\text { Yildirim } \\
\text { OA }\end{array}$ & 2021 & 33635508 & PMC7907665 & LOW & LOW & LOW & LOW & LOW & LOW & LOW & LOW \\
\hline
\end{tabular}

Table 2 Abbreviations: Domain 1 (D1): Risk of bias due to confounding; Domain 2 (D2): Risk of bias in selection of participants; Domain 3 (D3): Risk of bias in classification of interventions; Domain 4 (D4): Risk of bias due to deviations from intended interventions; Domain 5 (D5): Risk of bias due to missing data; Domain 6 (D6): Risk of bias in measurement of outcomes; Domain 7 (D7): Risk of bias in selection of the reported result. 
According to the first domain, the risk of bias due to confounding was low for five studies and was moderate for two studies. Risk of bias regarding the selection of participants classified all studies as low. For the risk of bias in the classification of interventions (domain three), five articles had a low risk, while two had a moderate risk of bias. The risk of bias due to deviations from intended interventions is classified low for all articles (domain four). There were six articles with a low risk of bias due to missing data, and one study had no information for bias assessment in the fifth domain. One study had a moderate risk of bias in outcomes' measurements, while the others had a low bias risk for the sixth domain. For the seventh domain, all studies had a low risk of bias concerning a selection of the reported result (Table 2, Appendix).

\section{Impact of the Pandemic on Cancer Palliative Care}

The effects on oncological palliative care were divided in two broad areas:

\section{Patient-related factors}

\section{Medical community}

Patient-related factors: The pandemic of COVID-19 affected several factors like quality of life, emotional functioning and mental well-being of the late-stage cancer patient. A German study evaluated cancer patient's quality of life of patients, and their relatives, during the first wave of the pandemic (04-07-2020) Troschel [10]. Brain tumor patients and their relatives experienced a reduction in quality of life, showing a significant increase in distress, anxiety, and depression. Cancer patients suffered from depressive symptoms more than their relatives $(p<0.001)$ and a decrease in their quality of life $(p<0013)$. Patients of advanced age, less physically active, ongoing therapy, poor living conditions, or lack of day job decreased overall quality of life. Social support (ten or more social contacts) increased well-being, reducing the risk of depression (70\%), anxiety (39\%), and distress (65\%) in comparison to that of patients experiencing social distancing Troschel [10].

Anxiety and depression increased during the pandemic of COVID-19 for a group of 595 cancer patients residing in Turkey Yildirim [11]. Disruption of treatment and related delays increased depression and anxiety levels, as measured by Beck Depression Inventory (BDI) and Beck Anxiety Inventory (BAI). This decrease in overall well-being was more pronounced for older cancer patients $(p=0.021)$ and female cancer patients $(p=0.000)$. Those patients using public transportation experienced more treatment delays $(p=0.038)$ due to its limited availability. Age was a factor related to a reduction in well-being, as older patients tend to reschedule their appointments due to fear of infection. Mental illnesses were more severe in Turkey areas in quarantine with strict governmental measures due to the COVID-19 lockdown Yildirim [11].

Cancer patients in India were also affected by the pandemic, reporting issues with their standard of care, according to a crosssectional study conducted by Mitra and collaborators Mitra [12]. A total of 36 patients responded to the poll, where the main challenges reported by them were an increase in anxiety (91.7\%), lack of support groups and psychological treatment (94.4\%), delays or rescheduling of nutritional appointments (88.9\%), issues with transportation to hospital facilities $(77.8 \%)$, restriction of visitors (72.2\%), and availability of chemotherapy slots in daycare (55.6\%). Other concerns were slot availability for consultation (41.7\%), network issues (33.3\%), maintaining precautionary measures (33.3\%), availability of chemotherapy medication
(22.2\%), rescheduling of radiotherapies and long waiting hours (22.2\%), rescheduling of tumor board meetings (19.5\%) and surgeries (13.8\%), and issues with visa extensions (11.2\%); Mitra [12]. According to Bartels et al., cancer patients experienced the rescheduling of their medical visits, impacting their current medical care Bartels [1]. This study conducted in the Netherlands stated the reasons for rescheduling were mainly fears regarding a potential infection with SARS-CoV-2, either from their contacts with physicians or by visiting health care facilities Bartels [1]. Feelings of isolation increased during the pandemic (from 18\% to 67\%). Moreover, the overall quality of life and emotional functioning for stage IV cancer patients with bone metastases decreased during the first lockdown of COVID-19 (quality of life from 72.4 to 68.7, emotional functioning from 83.6 to 79.2 , all $p<0.01$ ); Bartels [1]. Even though the previous changes are statistically significant, they are very small.

Medical community: According to the outcomes of this report, factors affected in the clinical setting entailed modifications in standards-of-care for cancer patients in palliative care, changes in policies for medical attention, and health infrastructure, with an observed increase in mental health disorders for patients and health care providers.Nieder and collaborators evaluated palliative radiotherapy (PRT) in the last 30 days of life of cancer patients before and during the pandemic. The study compared PRT received pre-COVID with those given during the pandemic. From 47 patients in the pre-COVID phase, $0.9 \%$ received PRT each business day, and $53 \%$ for bone metastases. Of 61 patients treated during the pandemic, $1 \%$ received PRT each business day, and $41 \%$ for bone metastases. These findings indicated a significant reduction in Norway's recommendations for PRT for bone metastases Nieder [13].

The capacity of palliative care services was reduced in MiddleEastern and North-African countries (MENA) according to the WHO International Health Regulations, as Boufkhed and collaborators showed in their cross-sectional online survey. Analyses of 43 palliative care services of 12 countries in MENA indicated modifications in palliative care services. $30 \%$ of the medical respondents of the online survey mentioned the policy of Do-notresucitate changed in $30 \%$ of the services and was unavailable in 23\% of MENA. Despite the availability of handwashing facilities at points of entry (98\% of MENA medical respondents), there were concerns about disinfectant products (37\%), soap (35\%), or running water $(33 \%)$. Most of the respondents used telemedicine $(86 \%)$ and had a list for contacting patients and personnel (93\%), but only $37 \%$ had the contacts of the patient's relatives. The respondents reported high anxiety about getting infected with SARS-CoV-2 (median score 8, on a 1-10 scale), but only half of MENA's services had a stress-management protocol (53\%); Boufkhed [14].

Another study by Boufkhed and collaborators indicated a reduction in the preparedness and capacity of palliative care services in African countries during the pandemic. This crosssectional online poll collected 83 respondents from 21 countries of the African Palliative Care Association. The 2005 International Health Regulations was the guideline for conducting this poll. Most of the services had a procedure for COVID-19 management or other infectious diseases (63\%). Respondents manifested concerns over accessing running water, soap, and disinfectant products (43\%, 42\%, and 59\%, respectively). They also expressed biosafety concerns for themselves or their staff (52\%). Two in every five services in Africa (41\%) reported no available additional personal protective equipment. The majority of services $(80 \%)$ 
had the capacity for telemedicine instead of in-site visits. Half of the services $(52 \%)$ had palliative care protocols for psychological support and symptoms' management Namisango [15].

\section{DISCUSSION}

The objective of this review is to report the main characteristics of the oncological palliative care, recognized by the medical community, during the COVID-19 era. All selected studies indicated an impact on oncological palliative care due to the COVID-19 pandemic. Cancer patients receiving palliative care were affected in their overall quality of life, including social support, increased mental disorders, and delays in medical treatment.

Boufkhed and collaborators pointed out protocol changes for palliative care services in the Middle-East and African countries Namisango [15]. Mitra [12] emphasized the challenges faced by the cancer patient, appointment delays, and impairments in receiving medical services compared to that of the pre-COVID era (Mitra et al., 2020). There was an increase in emotional dysfunction, including high anxiety levels Mitra [12], and a reduction in the overall quality of life for both patients and their relatives Bartels [1]; Troschel [10]; Yildirim [11]. Patients manifesting anxiety and depression also experienced delays, rescheduling, or cancellations in their medical appointments and interventions. The outcomes of this rapid review suggest oncological palliative care was impaired and reduced during the pandemic of COVID-19. Prior research evaluated quality of life in developing countries for older cancer patients in palliative care, where sociodemographic characteristics (like age, gender, education, culture, and marital status) influenced life's quality Gayatri [16]. However, this systematic review comprehended studies between January 1990 and February 2019. For this reason, there is no information in this study about the impact of the COVID-19 on palliative care. To our knowledge, the present review is the first one reporting factors of oncological palliative care affected during this health crisis.

\section{Strengths and Limitations}

The present review has two main strengths. First, studies selected were those with high quality (ROBINS-I). Second, the samples of each study were representative, and as a consequence, the identified factors were meaningful. There were two major limitations in this work. The first one is that only one database was revised (PubMed), and we cannot discard the potential exclusion of research articles published in gray literature or other available databases. The second is that there were different selection criteria in the studies selected, which increased the variability of the outcomes. For those reasons, the reliability of the conclusions is limited.

\section{CONCLUSION}

The medical services for oncological palliative care were compromised during this health crisis. The extensive demand for medical attention affected doctors, nurses, and resources in the health sector. Consequently, the quality of health services for cancer patients was reduced in comparison to the pre-COVID era. Overall quality of oncological palliative care diminished, and this was observed with the rescheduling of visits or treatment because of patients' fears of getting infected with SARS-CoV-2. Mental health was affected, increasing anxiety and emotional dysfunction among patients, their relatives, and health staff. Scarcity in medical resources impacted low and middle-income countries more than that of high-income countries. Overall, more research is required to address the unmet needs of oncological palliative care. Identifying gaps in the standard of care may aid in developing interventions for late-stage cancer patients in the post-COVID era.

\section{ACKNOWLEDGEMENT}

We want to thank University Kino for the support provided for this work. Special thanks to Dr. Oscar Fernando Almazan-Holguin, who was a supporter of this research project. No funding agencies were involved in this research work.

\section{REFERENCES}

1. Bartels MMTJ, Gal R, van der Velden JM, Verhoeff JJC, Verlaan JJ, et al. (2021) Impact of the COVID-19 pandemic on quality of life and emotional wellbeing in patients with bone metastases treated with radiotherapy: a prospective cohort study. Clin Exp Metastasis 38(2): 209-217.

2. Sigorski D, Sobczuk P, Osmola M, Kuć K, Walerzak A. et al. (2020) Impact of COVID-19 on anxiety levels among patients with cancer actively treated with systemic therapy. Esmo Open 5(5): e 000970.

3. Gupta R, Sarma R, Mishra S, Bhatnagar S (2020) Rendezvous of cancer patients with logistic and socioeconomic challenges during COVID-19 pandemic: A case series. Indian Journal of Palliative Care 26 (Suppl 1): S156-S159.

4. Bakhribah H, Zeitouni M, Daghistani RA, Almaghraby HQ Khankan AA, et al. (2020) Implications of COVID-19 pandemic on lung cancer management: A multidisciplinary perspective. Crit Rev Oncol Hematol 156: 103120 .

5. Aapro M, Lyman GH, Bokemeyer C, Rapoport BL, Mathieson N, et al. (2021) Supportive care in patients with cancer during the COVID-19 pandemic. ESMO Open 6(1): 100038.

6. Pathania AS, Prathipati P, Abdul BAA, Chava S, Katta SS, et al. (2021) COVID-19 and cancer comorbidity: Therapeutic opportunities and challenges. Theranostics 11(2): 731-753.

7. Sterne JAC, Higgins JPT, Elbers RG, Reeves BC et al. (2016) Risk of bias in non-randomized studies of Interventions (ROBINS-I): detailed guidance.

8. Amin MB, Greene FL, Edge SB, Compton CC, Gershenwald JE, et al. (2017) The Eight Edition AJCC Cancer Staging Manual: Continuing to build a bridge from a population-based to a more "personalized" approach to cancer staging. CA Cancer J Clin 2017 67(2):93-99.

9. World Health Organization (2013) WHO Definition of Palliative Care.

10. Troschel FM, Ahndorf F, Wille LM, Brandt R, Jost J, et al. (2021) Quality of life in brain tumor patients and their relatives heavily depends on social support factors during the COVID-19 pandemic. Cancers (Basel) 13(6): 1276.

11. Yildirim OA, Poyraz K, Erdur E (2021) Depression and anxiety in cancer patients before and during the SARS-CoV-2 pandemic: association with treatment delays. Qual Life Res 26: 1-10.

12. Mitra M, Basu M (2020) A Study on Challenges to Health Care Delivery Faced by Cancer Patients in India During the COVID-19 Pandemic. J Prim Care Community Health 11: 2150132720942705.

13. Nieder C, Haukland CE, Mannsaker B, Yobuta R (2021) Palliative radiotherapy during the last month of life: Have COVID-19 recommendations led to reduced utilization? In Vivo 35(1): 649-652.

14. Boufkhed S, Harding R, Kutluk T, Husseini A, Pourghazian N, et al. (2021) What is the preparedness and capacity of palliative care services in Middle Eastern and North African countries to respond to COVID-19? A rapid survey. J Pain Symptom Manage 61(2): e13-e50.

15. Boufkhed S, Namisango E, Luyirika E, M Fam Med, Sleeman KE, Costantini M, et al. (2020) Preparedness of African Palliative Care Services to Respond to the COVID-19 Pandemic: A Rapid Assessment. J Pain Symptom Manage 60(6): e10-e26.

16. Gayatri D, Efremov L, Kantelhardt EJ, Mikolajczyk R (2021) Quality of life of cancer patients at palliative care units in developing countries: Systematic review of the published literature. Quality of Life Research 30: 315-343. 